\title{
La educación médica durante la contingencia sanitaria por COVID-19: lecciones para el futuro
}

\author{
Medical Education during the health contingency by COVID-19: Lessons for the future \\ Carlos R. Sierra-Fernández ${ }^{1 *}$, Mauricio López-Meneses ${ }^{2}$, Francisco Azar-Manzur ${ }^{3}$ y \\ Sergio Trevethan-Cravioto ${ }^{4}$ \\ ${ }^{1}$ Dirección de enseñanza; ${ }^{2}$ Coordinación de Educación Médica Continua; ${ }^{3}$ Coordinación de Posgrado; ${ }^{4}$ Subdirección de Enseñanza de Pregrado. \\ Instituto Nacional de Cardiología Ignacio Chávez, Ciudad de México, México
}

\section{Introducción}

La sociedad contemporánea vive en un cambio permanente y un avance vertiginoso en la generación del conocimiento. Los avances en la informática, en computación y en las tecnológicas de la información y comunicación (TIC) han potenciado su desarrollo.

Los problemas sociales exigen conocimiento de utilidad casi inmediata. Actualmente los problemas medioambientales, de cambio climático, de desigualdad económica y los de salud poblacionales graves han sido un reto gigante para todos los países para organizaciones públicas y privadas, y están condicionando nuevas circunstancias epidemiológicas: la mayor incidencia de enfermedades cronicodegenerativas, la aparición de nuevas enfermedades y la reactivación de algunas que ya se consideraban erradicadas".

La pandemia por el coronavirus 2 del síndrome respiratorio agudo grave (SARS-CoV-2), que provoca la enfermedad por coronavirus 2019 (COVID-19), ha trastocado todo el orden mundial, en todos los niveles. Es un problema de salud pública sin precedente que ha obligado al aislamiento social y a la detención de la actividad productiva y de la actividad educativa en todos los niveles. Se estima que más de 1.5 billones de estudiantes que acuden a escuelas y universidades fueron afectados por el cierre en 188 países hasta la primera semana de abril del 2020 (Organización de las Naciones Unidas para la Educación, la Ciencia y la Cultura) $)^{2}$.

Por su magnitud y gravedad, la pandemia por la COVID-19 necesita generar conocimiento de aplicación práctica inmediata y que el personal médico y paramédico esté preparado y actualizado con información teórica y práctica para atender a los pacientes y poder trabajar en forma coordinada como un equipo multidisciplinario ${ }^{3}$.

En este artículo se analizará cuál es el estado actual de la educación virtual, cómo ha sido la respuesta durante la pandemia COVID-19 y cómo se transformará la educación médica en todos los niveles y la formación médica continuada.

\section{¿Qué es la educación médica?}

Las educación médica se puede conceptualizar como la adquisición y desarrollo de conocimientos, habilidades y destrezas que ocurren durante las diferentes etapas de formación de médicos generales y especialistas. Esta incluye el ingreso a la carrera de medicina, la licenciatura, el posgrado en especialidades, los programas de maestría y doctorado, y
Correspondencia:

${ }^{*}$ Carlos R. Sierra-Fernández

E-mail: drsierra@ cardios.mx
Fecha de recepción: 22-04-2020

Fecha de aceptación: 28-04-2020 DOI: 10.24875/ACM.M20000073
Disponible en internet: 26-05-2020 Arch Cardiol Mex. 2020;90(Supl):50-55 www.archivoscardiologia.com 1405-9940 / @ 2019 Instituto Nacional de Cardiología Ignacio Chávez. Publicado por Permanyer. Este es un artículo open access bajo la licencia CC BY-NC-ND (http://creativecommons.org/licenses/by-nc-nd/4.0/). 
permanece toda la vida en lo que se denomina la educación médica continua.

El aprendizaje de la medicina es complejo, porque implica muchas habilidades teórico-prácticas, dentro de un marco humanista y ético. Como comenta el Dr. Lifshifz acerca de la innovación y creatividad en la educación médica, la educación tradicional de los médicos tiene tendencia a la superestabilidad, es decir, a la inercia que la hace resistente al cambio, lo cual ocasiona anacronismos, como formar médicos para circunstancias pasadas. Por esto es necesario un sistema educativo adaptativo y flexible que responda a los cambios mediante la innovación ${ }^{4}$.

Sabemos que el conocimiento médico cambia a una velocidad vertiginosa y si los médicos no tienen las habilidades para actualizarse y renovarlo de forma continua, rápidamente quedarán obsoletos ${ }^{5}$.

\section{Respuesta de la comunidad médica}

La aparición de un nuevo virus, el SARS-CoV-2, que originó una enfermedad nueva con evolución acelerada a una pandemia, con múltiples manifestaciones sistémicas, ha suscitado numerosos interrogantes en cuanto su patofisiología y complicaciones, y aún no se cuenta con un tratamiento específico y eficiente. La respuesta de la comunidad médica ha sido sorprendente para actualizar el conocimiento que se publica de diferentes formas, todos los días, con minutos de diferencia y que en muchos casos tiene una utilidad inmediata para la toma de decisiones en pacientes críticamente enfermos. Además de que se debe conciliar con investigación de rápida ejecución, para continuar generando conocimiento ante una emergencia sin precedentes ${ }^{6}$.

\section{Desarrollo e implementación de las tecnologías de la información}

En las últimas dos décadas ha ocurrido un avance en el desarrollo y aplicación de las TIC para la educación médica y la comunicación. En nuestro país su desarrollo ha sido muy heterogéneo, dependiendo de las ciudades en que se realiza y el ámbito de formación médica (universidades públicas, privadas y hospitales).

El acceso a la tecnología en dispositivos móviles o computadoras favorece su utilización. Esto es concomitante con un gran desafío, porque no hay un acceso generalizado de toda la comunidad médica o instituciones formadoras a la tecnología de vanguardia.
Esta situación de extrema gravedad en salud pública para todo el planeta ha obligado a la implementación acelerada de educación virtual en todos los niveles educativos, incluso desde la educación básica. Todas las instituciones educativas y hospitales han adoptado clases virtuales, seminarios web, tutoriales y la consulta de bibliotecas digitales para continuar con la actividad académica ${ }^{3,7}$.

Cada médico o profesional de la salud en forma cotidiana, momento a momento, ha utilizado en su entorno de aprendizaje personal el uso de redes sociales (Facebook, Twitter), la consulta de noticias y medios de información, chats con colegas, foros de discusión y seminarios web de diferentes expertos o sociedades científicas. Las webs de consulta de revistas han liberado los artículos de investigación de alto impacto, como el New England Journal of Medicine, Lancet 0 JAMA.

\section{Encuesta en línea en estudiantes y profesionales de la salud en México}

Con el objetivo de conocer y evaluar la opinión de los estudiantes que reciben diversos contenidos educativos en línea como parte del proceso académico que ha sido impactado por las medidas de distanciamiento social impuestas ante la pandemia, realizamos una encuesta en línea a 228 profesionales de la salud, matriculados en programas académicos formales en México. Los participantes invitados a contestar este instrumento fueron estudiantes de medicina en cursos clínicos (12.3\%), médicos internos de pregrado (3.9\%), médicos generales en preparación para el Examen Nacional de Aspirantes a Residencias Médicas ENARM 2020 (27.2\%), médicos residentes (46.5\%) y médicos especialistas cursando algún programa de educación médica continua (10.1\%). La distribución de los encuestados se resume en la figura 1.

En este grupo de personas evaluadas, el $98.7 \%$ de los encuestados afirmó estar recibiendo algún tipo de educación a distancia como alternativa a la suspensión de actividades académicas presenciales. La mayor parte de los que han recibido educación a distancia lo han hecho por medio de videoconferencias, siendo la plataforma Zoom (de la empresa Zoom Video Communications INC) la más utilizada, seguida de Google Meet, Webex y Skype. Cabe resaltar que aunque la mayor parte de los encuestados recibe clases a distancia, el modelo de enseñanza no se ha modificado, siendo las clases magistrales en formato de presentación de diapositivas el más empleado. Esto refleja que 
Grado académico del alumno:

230 respuestas

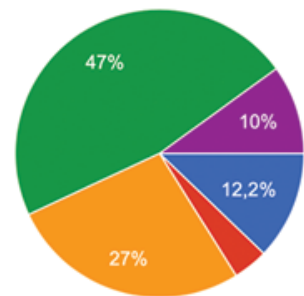

Estudiante de Medicina

Médico Interno de Pregrado

Médico General

Médico Residente

Médico Especialista

Como parte de tu programa académico formal, en estas últimas semanas de contingencia, ¿Has empleado alguna estrategia de educación a distancia? 230 respuestas

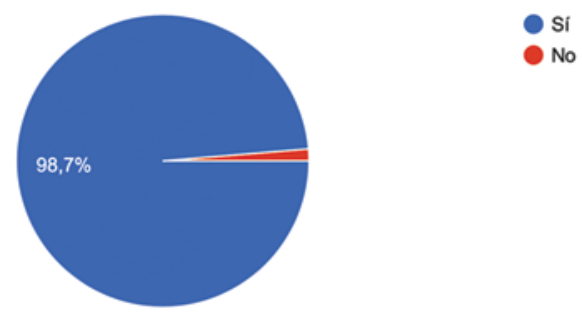

¿Qué plataforma has empleado en estos dias para recibir clases a distancia? 230 respuestas

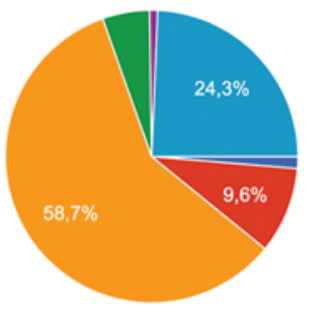

Skype

Google Meet / Hangout

Zoom

Webex

Go to meeting

Otra

Figura 1. Resultados de la encuesta en línea a 228 profesionales de la salud, matriculados en programas académicos formales en México. Grado académico y plataforma utilizada.

el cambio en el modelo de entrega de contenido educativo ha migrado a un medio digital pero no ha cambiado la estrategia docente. La mayor parte de los casos los alumnos reciben una videoclase, pero no necesariamente en todos se tiene un seguimiento periódico del progreso, ni un medio para retroalimentación y trabajo colaborativo. En esta encuesta se reporta que el $79.3 \%$ de los alumnos participa en algún gestor colaborativo del aprendizaje, siendo la estrategia Google Classroom la más empleada, seguida por Moodle y Blackboard (Fig. 1).

La experiencia del alumno en las clases a distancia es en general buena, en la figura 2 se expresan las opiniones sobre este medio en comparación con los formatos presenciales. Destaca que el $65.4 \%$ de los encuestados considera que su experiencia con medios a distancia es al menos igual o incluso mejor que las opciones disponibles de manera presencial. Sin embargo, la principal limitación sigue siendo la interacción entre docente y alumno, que mejora sustancialmente en las sesiones sincrónicas en tiempo real, pero que a decir de las personas encuestadas no sustituye al contacto humano.

Una ventaja considerable de las sesiones tipo clase grabada es la capacidad para reproducir el contenido a demanda. Esto permite al alumno consultarlo de manera libre y repetirlo tantas veces como sean necesarias para poder comprender los conceptos. Así mismo, la estrategia de grabación con respaldo en línea permite que este contenido esté disponible para consulta 
Con respecto a las clases tradicionales, ¿Cómo calificarias tu experiencia en clases grabadas o videoconferencia?

230 respuestas

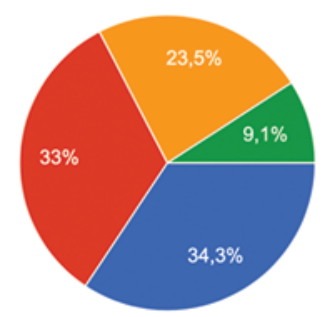

Mucho mejor

¿Cuál es la principal limitante de las clases a distancia tipo videoconferencia en tiempo real? 230 respuestas

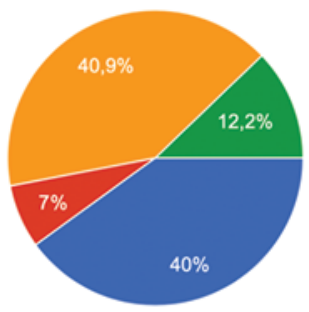

Poca interactividad con el profesor y compañeros

- Acceso a recursos tecnológicos

Menor capacidad para concentrar la atención en la clase en un entorno no escolarizado

El uso de diapositivas como medio único de explicación

¿Cuál es la principal ventaja de las clases a distancia grabadas? 230 respuestas

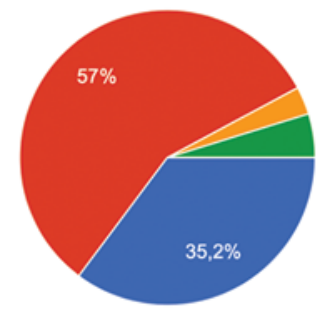

Disponibilidad en todo momento

- Capacidad de pausar, repetir o adelantar segmentos para reforzar $\mathrm{e}$ aprendizaje

Contar con el acervo histórico de las sesiones

Capacidad de compartir el contenido

Principal ventaja de las clases tipo videoconferencia en tiempo real 230 respuestas

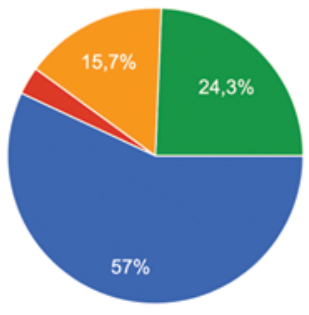

Posibilidad de hacer preguntas al profesor en tiempo real

Interacción con los compañeros

Mejora la atención de la audiencia al tener interactividad

Establecer un horario fijo y especifico para la clase

Figura 2. Resultados de la encuesta en línea a 228 profesionales de la salud, matriculados en programas académicos formales en México. Experiencia, ventajas y limitaciones.

durante actividades asistenciales, que en el caso de la formación médica enriquece el proceso de aprendizaje teórico y práctico. El tener la capacidad de consultar y consumir el contenido educativo sin barreras físicas ni temporales potencia la educación del médico con limitación importante de horario. 


\section{¿Cómo se consolidará la educación virtual?}

El cuestionamiento que surge con esta experiencia sin precedentes es cómo se transformará la educación y el aprendizaje médico cuando ya se haya controlado la pandemia. Existe evidencia en la literatura y como se refleja en los resultados de esta encuesta, de que la educación virtual para los médicos y personal sanitario es tan efectiva como los medios tradicionales (presencial en aula o semipresencial). Sin embargo, la educación en línea debe sustentarse en una metodología eficiente, con principios pedagógicos efectivos y contar con la tecnología necesaria8.

Para evitar una experiencia de aprendizaje virtual de poca calidad deben tenerse en cuenta algunos lineamientos básicos: a) habilidad para comprometer a los estudiantes en un ambiente de aprendizaje virtual, con una interacción intuitiva; b) favorecer la comunicación y conexión del profesor con los estudiantes; c) retroalimentación y apoyo durante el aprendizaje, que puede ser en tiempo real o asincrónico; d) utilizar vídeos o presentaciones breves (5-7 minutos) de gran calidad con conceptos bien definidos para mantener la atención y evitar el abandono de la actividad, y e) emplear herramientas dinámicas como mini-quiz, casos clínicos, foros de discusión y tecnología interactiva. En la encuesta realizada los participantes anotaron que la limitación principal fue la falta de interacción y retroalimentación, y que sin duda el contacto con el profesor es una motivación importante ${ }^{9}$.

\section{Utilidad de la telemedicina}

Otra estrategia de aprendizaje es la utilización de la telemedicina, no solo como herramienta para brindar consulta a los pacientes, ajustar tratamiento, prevenir complicaciones y evitar visitas innecesarias a los hospitales. Es útil para estudiantes de medicina que en la circunstancia de la emergencia sanitaria no pueden tener contacto clínico directo, pero que pueden entrevistar, dar orientación a los pacientes y aprender supervisados por un clínico con experiencia ${ }^{10}$.

Es interesante la experiencia reportada por los estudiantes de la universidad de Harvard, que utilizando la gran potencialidad de las redes sociales pudieron conformar grupos de trabajo para dar apoyo voluntario a comunidades vulnerables, así como elaborar herramientas de educación para la salud para médicos o público en general ${ }^{11,12}$.

\section{Formación docente en educación virtual}

Debe enfatizarse que los médicos y profesionales que se dediquen a la formación médica virtual o a distancia deben tener una formación específica y tener dominio de las plataformas educativas. Las funciones del tutor a distancia son relevantes dentro del proceso educativo en esta modalidad. Deben conocer los contenidos y tener conocimiento del modelo educativo virtual, saber de estrategias de enseñanza innovadoras y poseer habilidades interpersonales que promuevan que los estudiantes sean responsables de sus propios ritmos y logros de aprendizaje autónomo. La actividad docente está presente desde la planeación y el diseño de contenidos y actividades de aprendizaje, y también en el seguimiento, la monitorización y la evaluación del aprendizaje. Debe evaluarse todo el proceso educativo igual que en la enseñanza presencial ${ }^{7,8}$.

\section{Conclusiones}

El aprendizaje a distancia apoyado en las TIC es una herramienta poderosa para optimizar el aprendizaje en cualquier etapa de la formación profesional y en especial en el proceso de educación médica continua. En esta emergencia sanitaria se ha catapultado su utilización y con la perspectiva del tiempo sabremos cuánto impacto tuvo en salvar vidas de pacientes y de todo el personal sanitario que han trabajado con valentía y determinación atendiendo a estos pacientes.

Entre los desafíos y complejidades se encuentran cómo identificar la información más valiosa y apegada al método científico, cómo descartar información sin el rigor científico que aparece en forma exponencial y cómo el estrés y la urgencia en la toma de decisiones puede modificar una conducta terapéutica de modo que incluso pueda ser perjudicial para los pacientes.

También sabremos de qué forma pueden ayudar para mejorar el sistema de salud, la investigación clínica y la traslacional, y mejorar la colaboración entre diferentes instituciones y países.

Sin embargo, debemos recapitular/reflexionar con más paciencia ya que llegue la calma y consolidar la educación virtual sobre principios pedagógicos, el método científico y modelos educativos bien estructurados. Asimismo, debe haber un uso ético, consciente y prudente de las TIC.

No se debe perder nunca el enfoque humanístico y la cercanía con nuestros pacientes y profesores. 
Es muy probable que el mayor legado de la pandemia sea la revolución en la educación digital en todos los niveles de formación y en especial en la educación médica.

\section{Financiamiento}

La presente investigación no ha recibido ayudas específicas provenientes de agencias del sector público, sector comercial o entidades sin ánimo de lucro.

\section{Conflicto de intereses}

Los autores declaran no tener conflicto de intereses.

\section{Responsabilidades éticas}

Protección de personas y animales. Los autores declaran que para esta investigación no se han realizado experimentos en seres humanos ni en animales.

Confidencialidad de los datos. Los autores declaran que han seguido los protocolos de su centro de trabajo sobre la publicación de datos de pacientes.

Derecho a la privacidad y consentimiento informado. Los autores han obtenido el consentimiento informado de los pacientes y/o sujetos referidos en el artículo. Este documento obra en poder del autor de correspondencia.

\section{Bibliografía}

1. Ayittey FK, Ayittey MK, Chiwero NB, Kamasah JS, Dzuvor C. Economic impacts of Wuhan 2019-nCoV on China and the world. J Med Virol. 2020;92:473-5.

2. Nicola M, Alsafi Z, Sohrabi C, Kerwan A, Al-Jabir A, losifidis C, et al. The socio-economic implications of the coronavirus and COVID-19 pandemic: A review. Int J Surg. 2020 Apr 16. doi: 10.1016/j.ijsu.2020.04.018. [Epub ahead of print]

3. Mian A, Khan S. Medical education during pandemics: a UK perspective. BMC Med. 2020;18(1):100

4. Sánchez Mendiola M, Lifshitz Guinzberg A, Vilar Puig P, Martínez González A, Varela Ruiz ME, Graue Wiechers E, editores. Educación Médica teoría y práctica. Ciudad de México, México: Elsevier España; 2015.

5. Emanuel EJ. The inevitable reimagining of medical education. JAMA. 2020;323:1127-8.

6. Keesara S, Jonas A, Schulman K. COVID-19 and health care's digital revolution. N Engl J Med. 2020 Apr 2. doi: 10.1056/NEJMp2005835. [Epub ahead of print]

7. Cook DA Dupras DM. A practical guide to developing effective web-base learning. J Gen Inter Med. 2004;19(6):698-707.

8. Gillett-Swan J. The challenges of online learning: Supporting and engaging the isolated learner. J Learn Design. 2017;10:20-30.

9. Martin F, Bolliger DU. Engagement matters: Student perceptions on the importance of engagement strategies in the online learning environment. Online Learn. 2018;22:205-22.

10. Moazzami B, Razavi-Khorasani N, Dooghaie Moghadam A, Farokhi E, Rezaei N. COVID-19 and telemedicine: Immediate action required for maintaining healthcare providers well-being. J Clin Virol. 2020;126:104345.

11. Gallager Th, Scheleyer AM. "We Signed Up for This!" - Student and trainee responses to the Covid-19 Pandemic. N Engl J Med. 2020 Apr 8. doi: 10.1056/NEJMp2005234. [Epub ahead of print]

12. Soled D, Goel S, Barry D, Erfani P, Joseph N, Kochis M, et al. Medical student mobilization during a crisis: Lessons from a COVID-19 Medical Student Response Team. Acad Med. 2020 April 8. doi: 10.1097/ ACM.0000000000003401. [Epub ahead of print] 